\title{
Sensor element for a metal-insulator-semiconductor camera system (MISCam)
}

\author{
R. Schwarz ${ }^{\text {a b,* }}$, M. Fernandes ${ }^{\text {a }}$, J. Martins ${ }^{a}$, A. Fantoni ${ }^{\text {a }}$, M. Vieira ${ }^{\text {a }}$, P. Sanguino ${ }^{\text {, }}$, \\ C.N. Carvalho ${ }^{\text {c }}$, T. Muschik ${ }^{\mathrm{d}}$ \\ a Electronics and Communications Department, Inst. Superior de Engenharia de Lisboa (ISEL), P-1949-014 Lisbon, Portugal \\ b Physics Department, IST, P-1049-001 Lisbon, Portugal

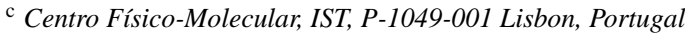 \\ ${ }^{\mathrm{d}}$ Nokia GmbH, D-89081 Ulm, Germany
}

Received 22 September 2003; received in revised form 26 March 2004; accepted 31 March 2004

\begin{abstract}
We discuss the operation of a new type of optical sensor (MISCam) based on a metal-insulator-semiconductor (MIS) structure. The operation principle relies on light-induced changes of the band bending and barrier height at the interface between semiconductor and insulator. An image is obtained from the quenching of the ac signal in analogy to the principle of the laser-scanned photodiode (LSP). Lateral resolution depends on the semiconductor material chosen. We have characterised the MIS structures by $C-V, I-V$, and spectral response measurements testing different types of insulators like a-Si $\mathrm{N}_{4}, \mathrm{SiO}_{2}$, and $\mathrm{AlN}$. The presence of slow interface charges allows for image memory. Colour sensors can be realised by controlling sign and magnitude of the electric fields in the base and the interface region. (C) 2004 Published by Elsevier B.V.
\end{abstract}

Keywords: MIS detector; Image sensor; $C-V$ curve; Spectral response

\section{Introduction}

Metal-insulator-semiconductor (MIS) structures based on hydrogenated amorphous silicon have been used by Jackson in 1989 to study metastable effects like light-induced defect formation [1] and defect kinetics, since the carrier density can be simply controlled by the gate bias, and defect density variations can be monitored easily by capacitance-voltage $(C-V)$ characteristics [2]. This is important for the evaluation of the performance of thin-film field-effect transistors (TFTs) which commonly employ a hydrogenated a-SiN insulator layer as gate dielectric. It could be shown by comparison of electron spin density measurements with the threshold voltage shift in $C-V$ characteristics under illumination that positive fixed charges, which are created in the bulk of the silicon nitride, are efficiently trapped at or near the insulator-semiconductor (i/s) interface while electrons are drifted to the gate electrode [3].

\footnotetext{
* Corresponding author. Tel.: +351-21-831-7289; fax: +351-21-831-7114.

E-mail address: rschwarz@isel.pt (R. Schwarz).
}

During a study of the influence of small leakage light on TFT performance Muschik et al. have measured a change of sign in the spectral response of a-SiN/a-Si double layers illuminated through a semitransparent gate contact by chopped light [4]. Under zero dc-voltage bias the ac-photocurrent signal was positive in the blue region and negative in the red spectral range as shown in Fig. 1(a). Applying a sufficiently large negative or positive bias voltage the spectral response curve could be shifted entirely to positive or negative values, respectively. In addition, we followed the shift of the zero crossing of the phase of the photocurrent at a given wavelength after applying a certain bias voltage under illumination as shown in Fig. 1(b). A first conclusion was to assume that the sign reversal in the ac-photocurrent at fixed wavelength was due to a change or even a reversal of the direction of the electric field in the a-Si:H layer near the insulator interface. The zero-crossing in the spectral response curve indicated that the space charge field, denoted by $\mathrm{F}$ in Fig. 2, was opposite to the field near the back contact (B in Fig. 2), and the main contribution to the ac-photocurrent was due to displacement currents in those two regions. The symbol $\Psi_{\mathrm{S}}$ denotes the band bending at the front interface $\mathrm{F}$. The direction of this band bending follows from the positive 

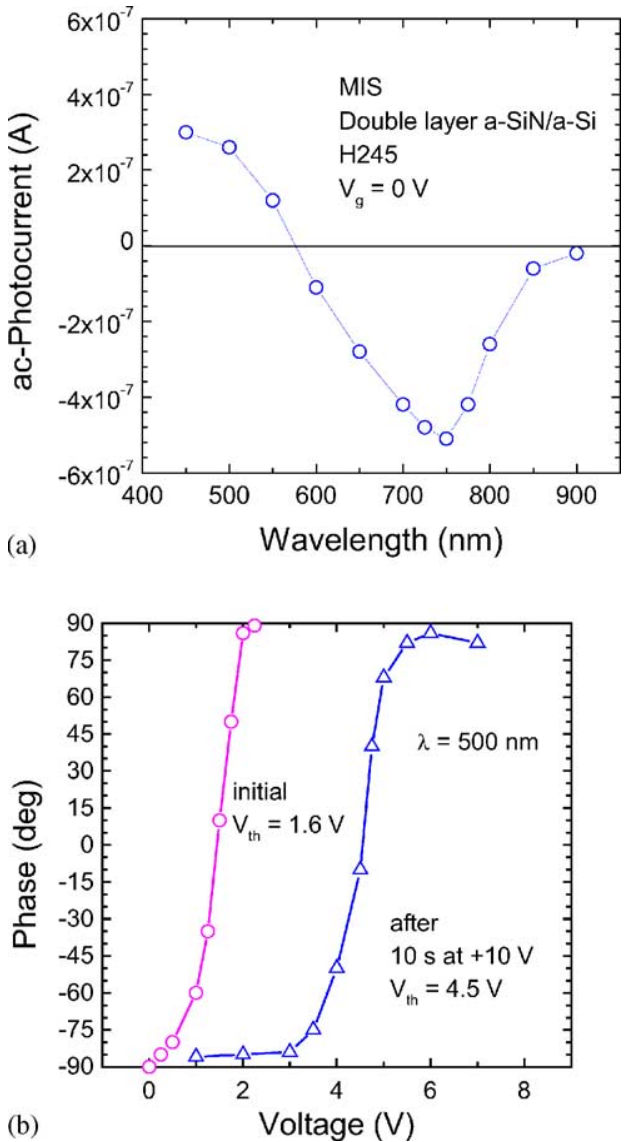

Fig. 1. (a) Crossover of spectral response in an $a-\mathrm{SiN}_{x}: \mathrm{H} / \mathrm{a}-\mathrm{Si}: \mathrm{H}$ MIS structure. (b) Shift of phase flip after voltage stress under illumination during 10 s (after Ref. [4]).

sign of the ac-photocurrent in the blue spectral region (see Fig. 1(a)). Light of this wavelength range is absorbed totally in region $\mathrm{F}$.

Now only a small step is needed to explain why such MIS structures have attractive features for image sensor applications. The ISEL group has recently shown that a-Si:H based p-i-n devices can be used for sensitive two-dimensional image read-out due to the local quenching of the trans-

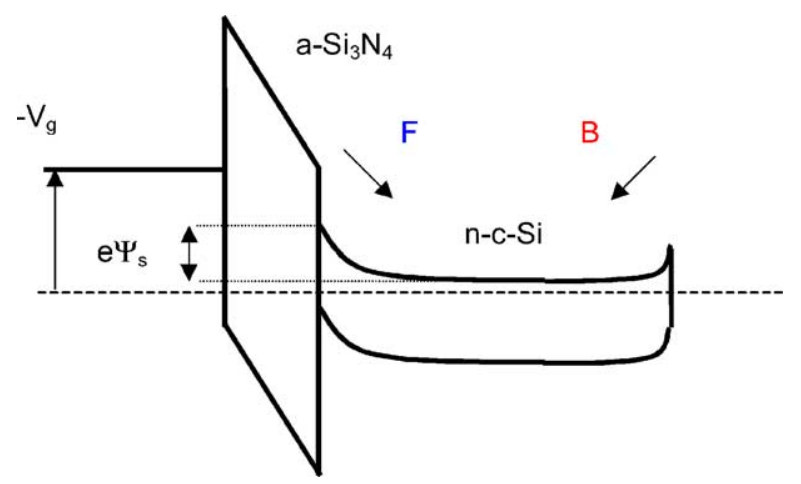

Fig. 2. Band diagram of a MIS structure under negative gate bias as deduced from the spectral response in Fig. 1. verse photovoltage under illumination. This principle is realised in the laser-scanned photodiode, LSP [5], where a low-intensity laser beam scans the detector surface and the image information is read out by the ac-component of the short-circuit current. Detailed studies were done by analysing $I-V$, spectral response, and $C-V$ curves, by testing the inclusion of highly resistive a-SiC:H alloy layers, and by monitoring memory effects after prolonged illumination [6,7]. In the MIS structure a similar quenching of the ac-photoresponse is expected under additional bias light illumination.

Several years ago, Fortunato et al. actually studied such MIS structures by measuring the photocharge after short light pulses under different bias conditions. They interpreted their results by numerical calculation of the surface potential at the $\mathrm{i} / \mathrm{s}$-interface and the field distribution inside the semiconductor film [8]. In this contribution we will consider MIS devices made of different gate contact materials (ITO, $\mathrm{Al}, \mathrm{Cr}$ ), different gate dielectrics like SiN and $\mathrm{AlN}$, and both monocrystalline and amorphous semiconductor base layers. We conclude that the operation of a MIS sensor is basically similar for all those material combinations. Differences are observed as far as signal height, signal speed, and hysteresis effects are concerned.

\section{Experiments}

\subsection{MIS sample structure}

The hydrogenated amorphous silicon and silicon-nitride layers in the tested structures were all deposited by usual PECVD processes. Some insulator layers were deposited on $\mathrm{n}$ - or p-type monocrystalline silicon. Gate contacts were prepared by thermal evaporation in the case of $\mathrm{Al}$ and $\mathrm{Cr}$ metals and by plasma-assisted evaporation of indium-tin-oxide powder in the case of ITO contacts.

The AlN insulator films were deposited by reactive sputtering in an $\mathrm{Ar} / \mathrm{H}_{2}$ plasma of an $\mathrm{Al}$ target [9] onto p-type crystalline silicon wafers with a doping concentration of about $10^{14} \mathrm{~cm}^{-3}$ to form MIS structures and on quartz glass substrates for reference measurements. The process pressure was held at $3 \mathrm{mTorr}$, the nitrogen flow at $10 \mathrm{sccm}$, and the dc plasma discharge current density was about $40 \mathrm{~mA} / \mathrm{cm}^{2}$. We have previously performed $I-V$ and $C-V$ measurements and obtained an estimate of the defect density of $10^{11}$ to $10^{12} \mathrm{~cm}^{-2} \mathrm{eV}^{-1}$ at an energetic level of $0.7-0.8 \mathrm{eV}[10]$.

\subsection{Measurement techniques}

Standard equipment was used for $I-V, C-V$, and spectral response measurements. In the case of low optical transmittance of the metal gate contacts we used a current-voltage converter followed by an averaging transient digitiser for time-resolved measurements and lock-in technique for the steady-state analysis. 

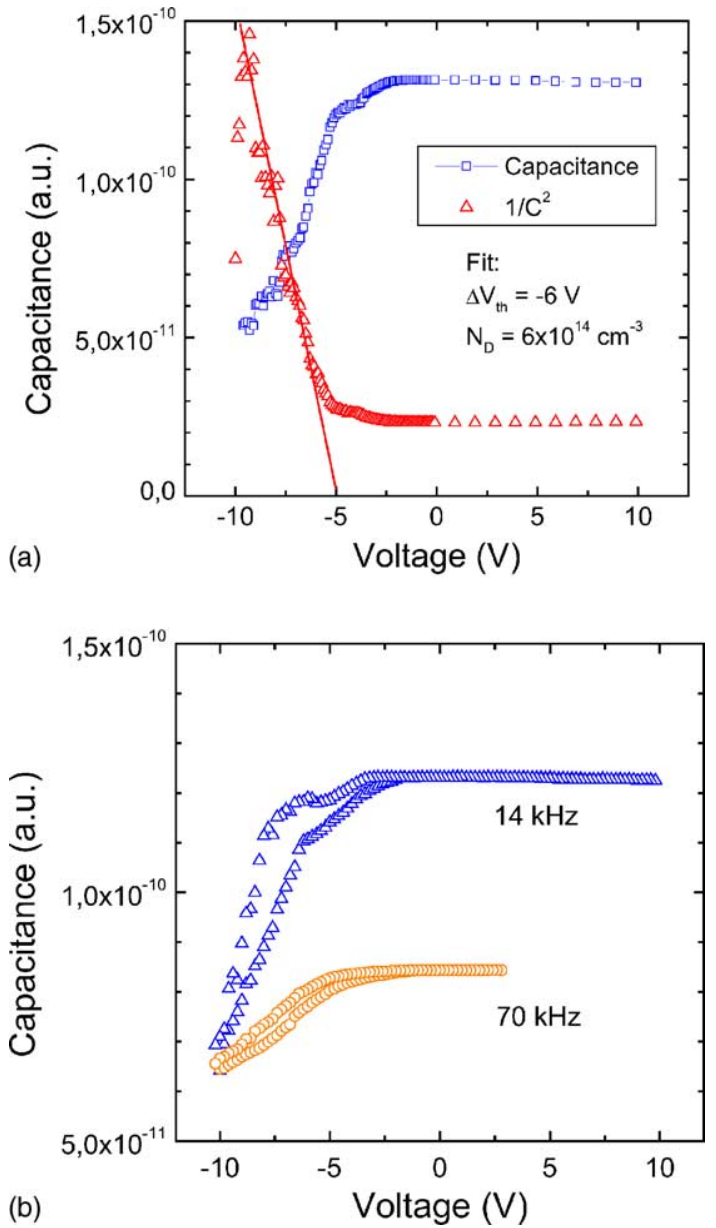

Fig. 3. $C-V$ curves of SiN MIS structure. (a) Linear fit to $1 / C^{2}$. (b) Frequency dependence and hysteresis.

\section{Single spot characterisation}

First we show the effect of interface charges in a silicon nitride MIS structure by employing techniques similar to the ones used by Fortunato et al., however, instead of measuring the photoinduced charge we rather looked, first, at the time decay of the sample capacitance, $C(t)$, up to the ms range [10], then at the shift of the quasi-dc $C-V$ curves, and, finally at hysteresis effects in the $C-V$ characteristic. The analysis of the $C-V$ curve in Fig. 3(a) leads to an estimate of the substrate doping level of about $6 \times 10^{14} \mathrm{~cm}^{-3}$, with very large interface charge densities of about $5 \times 10^{12} \mathrm{~cm}^{-2}$ derived from the threshold voltage shift. More information is obtained from Fig. 3(b). The change in slope is consistent with the existence of fast and slow states at or near the interface. At low frequency of $14 \mathrm{kHz}$ a small dip is seen when the voltage is scanned towards negative values, indicating reemission of trapped charges at a given level of band bending. At $70 \mathrm{kHz}$ the hysteresis is much less pronounced. In analogy to conclusions from our earlier study in a-Si:H based p-i-n LSP sensors, the large threshold voltage shift could be exploited for image memory purposes [7].

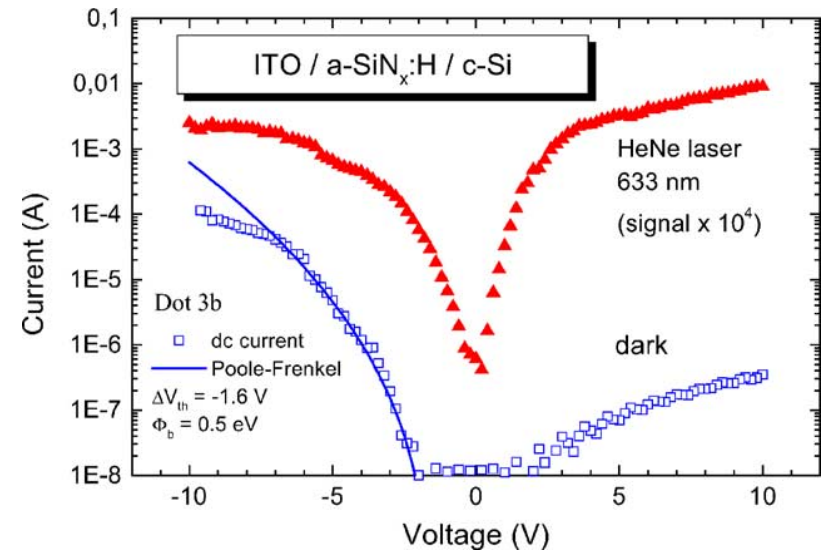

Fig. 4. Dark and photocurrent as a function of $V_{\mathrm{g}}$ in SiN MIS. The full line is a fit to the Poole-Frenkel ansatz.

Fig. 4 shows the $I-V$ characteristics in dark and under illumination with bias light from a HeNe laser. In dark we again obtain a large threshold voltage shift, similar to the $C-V$ results. We tried to fit the $I-V$ curve with a Poole-Frenkel ansatz [11]. The good fit hints to transport via traps in a-SiN:H with an average energy depth of $0.5 \mathrm{eV}$.

Fig. 5 shows the spectral response of the ac-readout of the MIS structure with a broad minimum near $750 \mathrm{~nm}$. This is in contrast to usual response curves in $\mathrm{p}-\mathrm{i}-\mathrm{n}$ diodes. The minimum observed here may stem form the existence of opposite fields that compensate contribution to ac-current for a certain generation profile. Detailed numerical analysis would be necessary to reproduce this behaviour in detail. What is important for the application as a sensor structure is the strong quenching effect seen for additional light bias as shown by the curve with lowest response values in Fig. 5 .

As far as bias voltage dependence of the spectral response curves is concerned we see an inverse behaviour for the SiN and the AlN samples, consistent with the opposite type of doping in the respective c-Si substrates.

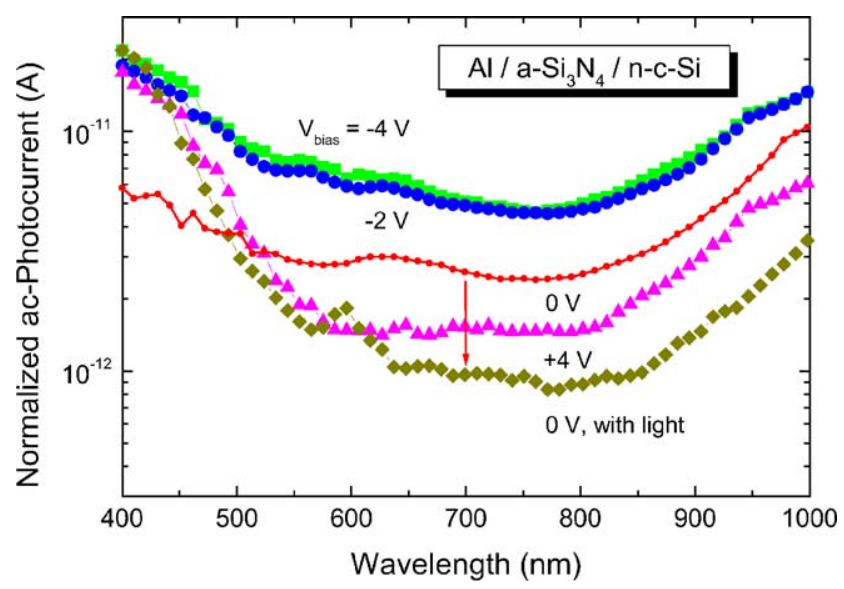

Fig. 5. Spectral response of the a-SiN on c-Si MIS structure for $V_{\mathrm{g}}=-4$, $-2,0$, and $+4 \mathrm{~V}$. The lowest curve is taken at zero bias under additional illumination with white light. The arrow indicates the photosignal quenching effect under illumination. 

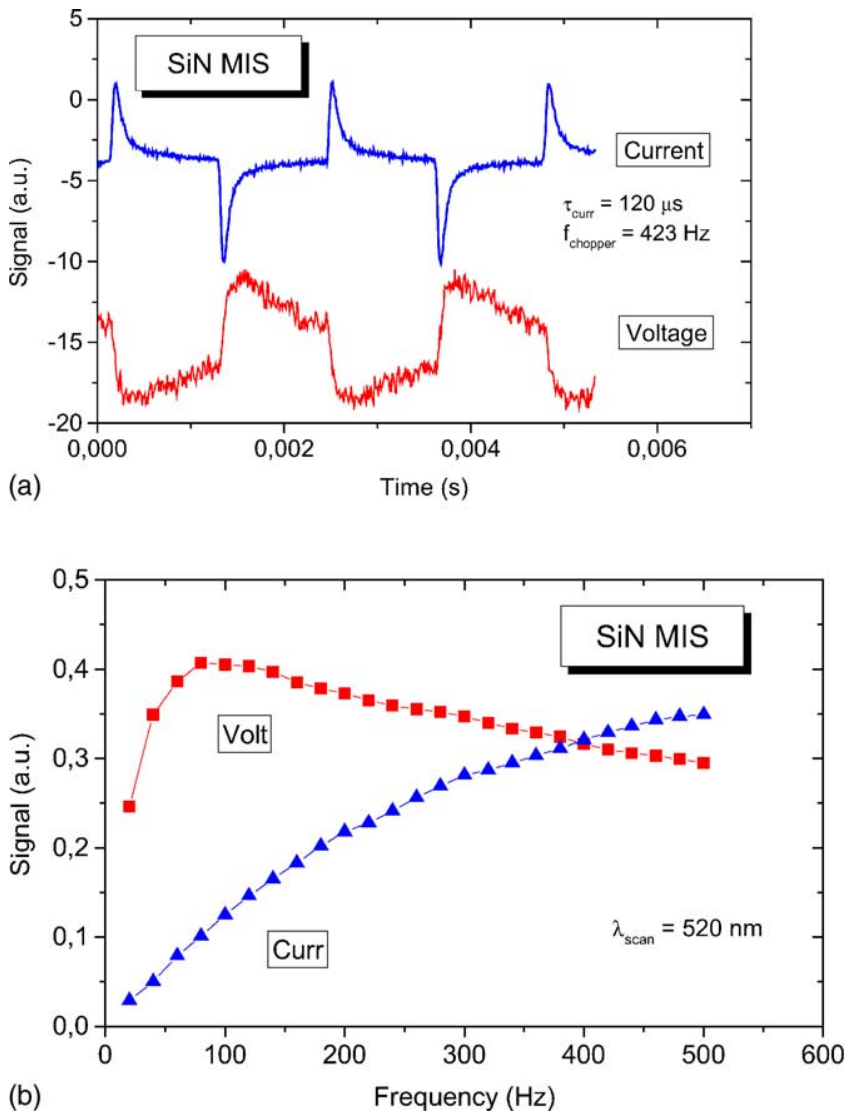

Fig. 6. ac-signal as a function of chopper frequency of a circular SiN MIS sensor element of $2 \mathrm{~mm}$ diameter. (a) Transients in the ac-readout of current and voltage at $423 \mathrm{~Hz}$. (b) ac-signal height as a function of chopper frequency.

Finally, we looked at the time-resolved behaviour of the ac-photocurrent in the a-SiN based structure. Fig. 6(a) reveals overshoots and undershoots in the current transients after switching of the light which, are compatible with the presence of the gate capacitor. Fig. 6(b) confirms zero signal for dc operation, and increase with frequency, as expected from an MIS detector. Since a mechanical chopper was employed in this study we have not reached the fastest possible transients in such structures.

\section{Image sensor}

In this section we looked at the two-dimensional readout of a $6 \mathrm{~mm} \times 6 \mathrm{~mm}$ large $\mathrm{Cr} / \mathrm{a}-\mathrm{SiN} / \mathrm{p}-\mathrm{c}-\mathrm{Si} / \mathrm{Al}$ sensor structure. A weak chopped solid-state laser spot of green colour $\left(\sim 100 \mu \mathrm{W} \mathrm{cm}{ }^{-2}\right.$ at $\left.530 \mathrm{~nm}\right)$ is scanned across the sensor and the ac-component is read out by lock-in amplifier. The image is a laser spot from a $633 \mathrm{~nm} \mathrm{HeNe}$ laser varying between 0.2 and $20 \mathrm{~mW} \mathrm{~cm}^{-2}$.

First, we see a decrease of the ac-signal with increasing image light intensity. Secondly, taking a $x-y$ scan of the illuminated area we find a spread of the laser beam image of about $1.5 \mathrm{~mm}$. No gate voltage was applied. Taking results from the work on a-SiC:H based image sensors into account, we expect to have a higher spatial resolution in MIS structures where the base layer consists of amorphous rather than monocrystalline or microcrystalline silicon [6].

As far as the selection of insulator material is concerned one would expect the lowest threshold voltage shift with silicon dioxide on c-Si due to its low interface defect density values achieved in thermally grown oxide films.

\section{Summary}

We have observed quenching of the ac-photocurrent signal in different MIS structures that can be used as 2D image sensors. The readout principle is similar to the LSP sensor. Large threshold voltage shifts occur in MIS sensors with a-SiN ${ }_{x}: \mathrm{H}$ insulator layers, whereas $\mathrm{AlN}$ represents a more stable gate dielectric. The experimental spatial resolution, which is controlled by lateral charge diffusion at the semiconductor-insulator interface, is about $1.5 \mathrm{~mm}$ in c-Si substrates and is expected to be below $50 \mu \mathrm{m}$ with a-Si:H based material. Colour separation is possible through modulation of the interface band bending by the external bias. Continuous recalibration is needed for image sensor application due to charge trapping under voltage bias and illumination occurring in the insulator layer or the insulator/semiconductor interface. On the other hand, the large threshold voltage shift can be used for temporary image storage.

\section{Acknowledgements}

We thank M. Bollu, Siemens AG, for supplying the a-SiN on c-Si substrate and A. Veloso and P.P. Freitas for supplying the AlN insulater layer. This work was financially supported by FCT project PRAXIS/P/EEI/12183/1998.

\section{References}

[1] D.L. Staebler, C.R. Wronski, Reversible conductivity changes in discharge-produced amorphous Si, Appl. Phys. Lett. 31 (1977) 292.

[2] W.B. Jackson, Defect kinetics in hydrogenated amorphous silicon MIS structures, Appl. Surf. Sci. 39 (1989) 273.

[3] J. Kanicki, D. Jousse, A. Gelatos, M.S. Crowder, Light-induced effects in hydrogenated amorphous nitrogen-rich silicon nitride films, J. Non-Cryst. Sol. 114 (1989) 612.

[4] T. Muschik, F. Wang, R. Schwarz, TU Munich, Unpublished, 1993.

[5] M. Vieira, M. Fernandes, J. Martins, P. Louro, A. Maçarico, R. Schwarz, M. Schubert, Laser scanned photodiodes (LSP) for image detection, IEEE Sens. J. 1 (2001) 158.

[6] M. Fernandes, M. Vieira, J. Martins, P. Louro, A. Maçarico, R. Schwarz, M. Schubert, Influence of the transducer configuration on the p-i-n image sensor resolution, Thin Solid Films 383 (2001) 65.

[7] R. Schwarz, P. Louro, Yu. Vygranenko, M. Fernandes, M. Vieira, M. Schubert, Memory effects in highly resistive $\mathrm{p}-\mathrm{i}-\mathrm{n}$ heterojunctions for optical applications, Thin Solid Films 403-404 (2002) 363. 
[8] G. Fortunato, L. Mariucci, C. Reita, V. Parisi, Experimental and theoretical evidence of space-charge photomodulation in metal/insulator/amorphous semiconductor structures, J. Non-Cryst. Sol. 114 (1989) 378.

[9] T.S. Plaskett, P.P. Freitas, N.P. Barradas, M.F. da Silva, J.C. Soares, Magnetoresistance and magnetic properties of NiFe/oxide/ co-junctions, J. Appl. Phys. 76 (1994) 6104.

[10] R. Schwarz, M. Niehus, S. Koynov, L. Melo, J. Wang, S. Cardoso, P.P. Freitas, Pulsed sub-band-gap photoexcitation of AlN, Diamond Rel. Mater. 10 (2001) 1326.

[11] J. Frenkel, On pre-breakdown phenomena in insulators and electronic semi-conductors, Phys. Rev. 54 (1938) 647.

\section{Biographies}

Reinhard Schwarz received a first degree in Physcis and Mathematics from the University of Stuttgart, Germany, in 1977, and a $\mathrm{PhD}$ degree from the University of Neuchâtel, Switzerland, in 1982. After his Post-doc at Princeton University, USA, he joined the Physics Department of the Technical University of Munich, Germany, between 1986 and 1996. There he headed a number of research projects in the field of amorphous silicon, a topic he continued since then in Portugal, first as an invited professor at the Instituto Superior Técnico, IST, Lisbon, then as Coordinator Professor at the Engineering Institute of Lisbon. Since 2003 he is Associate Professor at the Physics Department at IST focussing on GaN-based semiconductor research.

Miguel Fernandes was born in Portugal in 1970. He graduated in Physics and Materials Engineering by the Faculty of Sciences and Technology from the New University of Lisbon in 1995. Currently he is Assistant Professor in Electronics Department of ISEL, Lisbon, Portugal and investigator in the group of Applied Research in Microelectronic Optoelectronic and Sensors-GIAMOS in the same institution.

João Martins graduated in Electrotechnical and Computer Engineering at the Technical University of Lisbon, Portugal, in 1988, and received a Master of Science degree at this institution in 1993. He was a research member of the Microelectronics Group of the Institute of Systems and
Computer Engineering, INESC, in Lisbon after 1987. Since 1996 he is a Professor at the Electronics Department of ISEL, Lisbon, and investigator in the group of Applied Research in Microelectronic Optoelectronic and Sensors-GIAMOS. His research and development experience lies in several fields, like computer-aided design for electronics, and for semiconductor devices and sensors.

Alessandro Fantoni was born in Rome (Italy) in 1966. He received a university degree in Applied Mathematics from the University of Camerino, Italy (1992), and a PhD in Material Science, Micro- and Optoelectronics from the New University of Lisbon, Portugal (1999). He currently teaches Semiconductor Physics in the Electronics, Telecommunications and Computer Department of the Engineering Institute of Lisbon. His research interests are related to numerical analysis and simulation of micro- and optoelectronic thin film devices.

Manuela Vieira was born in Lisbon, Portugal. In 1986 she received the Master of Science in Solid State Physics-Microelectronic and in 1993 the $\mathrm{PhD}$ in Semiconductor Materials, both from the New University of Lisbon. She is now Coordinator Professor in Electronics in the Electronics and Communications Department, ISEL, Lisbon, Portugal. Currently she is the head of the Group in Applied Research in Microelectronic Optoelectronic and Sensors-GIAMOS, ISEL. She has several scientific papers and more than 15 years of experience in the field of thin films and devices. Her research activities have been mainly related to the development of optical sensors.

Pedro Sanguino has received his degree of Physics Engineering of the Instituto Superior Técnico, IST, Lisbon, Portugal, in 1999. After a 1-year period as project assistant in the field of amorphous silicon based solar cells he joined the $\mathrm{PhD}$ program at IST and is currently working on pulsed-laser deposition of thin film GaN.

Thomas Muschik received his diploma degree in Technical Physics of the University of Munich, Germany, in 1987. He joined the group of R. Schwarz to work towards a $\mathrm{PhD}$ in the field of photoluminescence of amorphous silicon. Currently he holds a position as project leader at Nokia company in Ulm, Germany, supervising the development and production of new models of mobile telephone systems. 